\title{
Study Family History of Psychiatry Disorders in Schizophrenia Patients
}

\author{
Arash Mowla ${ }^{a}$, , Sahar Bahrami ${ }^{\mathrm{a}}$
}

\begin{abstract}
Background: Schizophrenia is a chronic heterogeneous mental disorder that often has debilitating long-term outcomes. Our aim in this study is to survey history of psychiatric disorders in first-degree relatives of schizophrenia patients and its association with the disease clinical and demographic profile.
\end{abstract}

Methods: In this retrospective study the hospital records of all schizophrenia patients that had been admitted in Ibn Sina Psychiatric Hospital from March 2018 to March 2019 were surveyed. Histories of any psychiatry disorders in the first-degree relatives of the schizophrenia patients were searched. The patients with positive family history were compared with those with negative family history in regard to age of onset, sex, negative symptoms, substance abuse and education level.

Results: Of 250 files that were studied, $62(24.2 \%)$ patients had family history of psychiatry disorders. Schizophrenia (10.8\%), schizoaffective disorder (7.2\%) and bipolar disorder (4.2\%) were the most common psychiatry disorders in first-degree relatives of schizophrenia patients. Male sex, lower age at onset, substance abuse, negative symptoms, and lower education were more frequently observed in schizophrenia patients with positive family history.

Conclusions: Our study demonstrated that family histories of schizophrenia, schizoaffective and bipolar disorder were higher in family history of schizophrenia patients than normal population. Furthermore, positive family history for psychiatric disorder is associated with worse prognosis in schizophrenia patients.

Keywords: Schizophrenia; Psychiatry disorders; Family history; Bipolar disorder; First-degree relatives

Manuscript submitted August 30, 2020, accepted September 9, 2020

Published online December 9, 2020

a Substance Abuse and Mental Health Research Center, Shiraz University of Medical Sciences, Shiraz, Iran

${ }^{b}$ Corresponding Author: Arash Mowla, Department of Psychiatry, Hafez Hospital, Shiraz University of Medical Sciences, Shiraz, Iran.

Email: mowlaar@gmail.com

doi: https://doi.org/10.14740/jnr631

\section{Introduction}

Schizophrenia is a severe, chronic, and heterogeneous mental disorder that often has debilitating long-term outcomes. Its lifetime prevalence rate is estimated to be approximately $1 \%$ worldwide in the adult population $[1,2]$. Elucidation of etiological factors of schizophrenia remains a major challenge for researchers. In the last two decades several genome-wide association studies (GWAS) have been performed to unravel genetic causes of the disease and the role of familial genetic contribution to the disease formation [3-5]. Simultaneously, hundreds or even thousands of single nucleotide polymorphisms (SNPs) were reported, which individually could explain only a small fraction of the genetic contribution to the disease; however, cumulative effect of these risk variants may offer a larger share in genetic architecture of disease [6].

Family history of schizophrenia is the most important risk factor for schizophrenia [7]. Their meta-analysis found that estimates for schizophrenia risk were eight-fold for firstdegree relatives of one proband with schizophrenia compared to healthy control probands and increasing to 11 -fold for first-degree relatives with two probands with schizophrenia [8].

Recent studies have shown that the family history of axis I psychiatry disorders such as bipolar disorders, major depressive disorder and anxiety disorders are higher in schizophrenia patients than normal population [9-11]. Therefore, it could be hypothesized that the definition of familial loading based on the presence or absence of psychosis in families of patients with schizophrenia should be broadened by focusing on psychiatric disorder in general irrespective of a specific diagnosis.

Several studies have shown positive family history of psychiatry disorders in schizophrenia patients is associated with poorer prognosis and outcome $[12,13]$. Younger age at onset and substance abuse are reported more in schizophrenia patients with positive family history of schizophrenia [14].

Studies about family history of psychiatric disorders in schizophrenia patients in Iranian population are rare. In this research, we have surveyed the history of psychiatry disorders among siblings, parents and offspring of a sample of Iranian inpatient schizophrenia patients. Furthermore, we will survey the association between family history for psychiatry disorders and the demographic and clinical profile of the disease. 
Table 1. Family History of Psychiatry Disorders in First-Degree Relatives of Schizophrenia Patients

\begin{tabular}{cl}
\hline & Number (\%) \\
\hline Schizophrenia patients & $250(100 \%)$ \\
-FHx for psychiatry disorders & $188(75.8 \%)$ \\
+FHx for psychiatry disorders & $62(24.2 \%)$ \\
\hline
\end{tabular}

FHx: family history.

Table 2. Psychiatry Disorders in First-Degree Relatives of Schizophrenia Patients

\begin{tabular}{ll} 
Psychiatry disorders & Number $\mathbf{( \% )}$ \\
\hline Schizophrenia & $27(10.8 \%)$ \\
Schizoaffective disorder & $18(7.2 \%)$ \\
Bipolar disorder & $12(4.8 \%)$ \\
Major depressive disorder & $4(1.6 \%)$ \\
Obsessive compulsive disorder & $2(0.8 \%)$ \\
\hline
\end{tabular}

\section{Materials and Methods}

Our research is a retrospective study. We surveyed the hospital records of all schizophrenia patients admitted in Ibn Sina Psychiatric Hospital from March 2018 to March 2019. Histories of any psychiatry disorder in the first-degree relatives of the schizophrenia patients were searched. The patients were diagnosed to have schizophrenia according to the Diagnostic and Statistical Manual of Mental Disorders, Fifth Edition (DSM-5) criteria by a board-certified psychiatrist. We looked for psychiatry disorders in just the first-degree relatives like father, mother brother, sister and children. The patients with positive family history for psychiatry disorders were compared with those with negative family history in regard to age of onset of schizophrenia, negative symptoms, sex, substance abuse and education. This research was approved by Shiraz University of Medical Sciences Review Board. It was approved by Shiraz Medical School ethical committee for human studies.

\section{Statistical analysis}

Obtained data were statistically analyzed with IBM SPSS Statistics 21.0 for Windows. Chi- Square and independent t tests were used, as appropriate, to compare the demographic and clinical characteristics of the two groups. P value less than 0.05 was considered as statistically significant.

\section{Results}

Of 250 files studied, $62(24.2 \%)$ patients had family history of axis I psychiatry disorders. Schizophrenia (10.8\%), schizoaffective disorder $(7.2 \%)$ and bipolar disorder $(4.8 \%)$ were the most common psychiatry disorders in first-degree relatives of schizophrenia patients. Tables 1 and 2 depict the family history of psychiatry disorders in schizophrenia patients.

Patients with positive family history had worse prognosis compared to their counterparts without positive family history (Table 3).

\section{Discussion}

To our knowledge this is the first report of family history of psychiatric disorders in Iranian schizophrenia patients. In line with previous studies [7-10], our research revealed that schizophrenia is the most common psychiatry disorder in firstdegree relatives of schizophrenia patients. The lifetime risk of schizophrenia in first-degree relatives of our patients $(10.8 \%)$ was near the lifetime risk of schizophrenia $(8.5 \%)$ in the first-

Table 3. Demographic and Clinical Data of Schizophrenia Patients

\begin{tabular}{llll}
\hline Schizophrenia & +FHx for psychiatry disorders & -FHx for psychiatry disorders & P value \\
\hline Female (\%) & 33 & 55 & 0.000 \\
Age onset (mean) & 18.45 & 25.17 & 0.000 \\
Substance abuse (\%) & 21 & 15 & 0.000 \\
Education (\% lower than diploma) & 51 & 24 & 0.000 \\
Negative symptoms (\%) & 20 & 6 & 0.000 \\
\hline
\end{tabular}

FHx: family history. 
degree relative of schizophrenia patients in some western studies [15]. This demonstrates that in different culture risk of developing schizophrenia in first-degree relatives of schizophrenia patients is high. The rate of developing schizophrenia was more for developing any other psychiatry disorder in relative of schizophrenia patients in our study. This shows some specification for genes in schizophrenia. Our findings did not support some previous researches demonstrating similar genetic vulnerability among psychiatry disorders $[11,16]$. In line with our findings, Jeppesen et al in their cohort study found that family history of psychotic mental disorders in contrast to non-psychotic psychiatry disorders were higher in schizophrenia patients [17].

Several researches have revealed early onset cases of schizophrenia are familial variant of the adult-onset schizophrenia and are associated with more family history of the disease $[18,19]$. Early-onset cases have demonstrated more enduring clinical morbidity and psychosocial disability [20, 21]. In line with these studies, we found that Schizophrenia cases with positive family history for schizophrenia had lower age at onset, more negative symptoms, more rates of substance abuse and lower education.

Negative symptoms of schizophrenia form a complex clinical constellation of symptoms that challenge both diagnosis and treatment. By definition, negative symptoms mean the absence of normal functions [22]. Negative symptoms in schizophrenia patients have been reported in familial cases more often and have been associated with poor outcome and more disability [23, 24]. Similarly, we observed schizophrenia patient with positive family history were more associated with negative symptoms and had poorer outcome.

Several studies have report that schizophrenia patients with positive family history have younger age at onset and are associated with more substance abuse [25-27]. Our results showed that schizophrenia patients with positive family history have lower age at onset, are more male, have lower education attainment and these patients abuse substance more frequently. Our findings are in keeping with the results of the previous studies.

At the end, it needs to be noted that our study is preliminary and larger studies with more patients are needed to confirm the results.

\section{Conclusions}

We found that $24.2 \%$ of our schizophrenia patients had family history of psychiatry disorders. Schizophrenia, schizoaffective and bipolar disorders were the most common psychiatry disorders in the family history of our patients. Our results showed that schizophrenia patients with positive family history were more associated with lower age at onset, male sex, substance abuse, negative symptoms and lower education.

\section{Acknowledgments}

None to declare.

\section{Financial Disclosure}

This study was supported by a grant from Shiraz University of Medical Sciences (92-01-01-7133) to Dr. Arash Mowla.

\section{Conflict of Interest}

All authors declare no conflict of interest.

\section{Author Contributions}

All the authors contributed to study designing, data collection, data analysis and writing the manuscript. Dr. Arash Mowla is the principal investigator of this research. All authors read and approved the final version of the manuscript.

\section{Data Availability}

The data supporting the findings of this study are available from the corresponding author upon reasonable request.

\section{References}

1. Lehman AF, Lieberman JA, Dixon LB, et al. "Practice guideline for the treatment of patients with schizophrenia", 2nd ed. American Psychiatric Association. 2010. Available at: https://psychiatryonline.org/pb/assets/raw/ sitewide/practice_guidelines/guidelines/schizophrenia. pdf.

2. Bergen SE, O'Dushlaine CT, Lee PH, Fanous AH, Ruderfer DM, Ripke S, International Schizophrenia Consortium SSC, et al. Genetic modifiers and subtypes in schizophrenia: investigations of age at onset, severity, sex and family history. Schizophr Res. 2014;154(1-3):48-53.

3. International Schizophrenia Consortium, Purcell SM, Wray NR, Stone JL, Visscher PM, O'Donovan MC, Sullivan $\mathrm{PF}$, et al. Common polygenic variation contributes to risk of schizophrenia and bipolar disorder. Nature. 2009;460(7256):748-752.

4. Schizophrenia Psychiatric Genome-Wide Association Study Consortium. Genome-wide association study identifies five new schizophrenia loci. Nat Genet. 2011;43(10):969-976.

5. Schizophrenia Working Group of the Psychiatric Genomics Consortium. Biological insights from 108 schizophrenia-associated genetic loci. Nature. 2014;511(7510):421427.

6. Bergen SE, Petryshen TL. Genome-wide association studies of schizophrenia: does bigger lead to better results? Curr Opin Psychiatry. 2012;25(2):76-82.

7. Mortensen PB, Pedersen MG, Pedersen CB. Psychiatric family history and schizophrenia risk in Denmark: which mental disorders are relevant? Psychol Med. 2010;40(2):201-210.

8. Le L, R K, B M, Mj G. Risk of schizophrenia in relatives 
of individuals affected by schizophrenia: A meta-analysis. Psychiatry Res. 2020;286:112852.

9. Lichtenstein P, Yip BH, Bjork C, Pawitan Y, Cannon TD, Sullivan PF, Hultman CM. Common genetic determinants of schizophrenia and bipolar disorder in Swedish families: a population-based study. Lancet. 2009;373(9659):234239.

10. Prasad KM, Sanders R, Sweeney J, Montrose D, Diwadkar V, Dworakowski D, Miewald J, et al. Neurological abnormalities among offspring of persons with schizophrenia: relation to premorbid psychopathology. Schizophr Res. 2009;108(1-3):163-169.

11. Ross RG, Compagnon N. Diagnosis and treatment of psychiatric disorders in children with a schizophrenic parent. Schizophr Res. 2001;50(1-2):121-129.

12. Kakela J, Nordstrom T, Haapea M, Jaaskelainen E, Miettunen J. Association between family history of mental disorders and outcome in psychotic disorders. Psychiatry Res. 2018;270:616-621.

13. Kakela J, Marttila R, Keskinen E, Veijola J, Isohanni M, Koivumaa-Honkanen H, Haapea M, et al. Association between family history of psychiatric disorders and longterm outcome in schizophrenia - The Northern Finland Birth Cohort 1966 study. Psychiatry Res. 2017;249:16-22.

14. Matzova Z, Skodacek I, Suba J, Bohmer F. Comparison of history of adolescents with substance-induced psychosis, early onset schizophrenia and substance use disorders. Bratisl Lek Listy. 2014;115(12):771-775.

15. Picchioni MM, Murray RM. Schizophrenia. BMJ. 2007;335(7610):91-95.

16. Peerbooms OL, van Os J, Drukker M, Kenis G, Hoogveld L, Mthfr in Psychiatry Group, de Hert M, et al. Metaanalysis of MTHFR gene variants in schizophrenia, bipolar disorder and unipolar depressive disorder: evidence for a common genetic vulnerability? Brain Behav Immun. 2011;25(8):1530-1543.

17. Jeppesen P, Larsen JT, Clemmensen L, Munkholm A, Rimvall MK, Rask CU, van Os J, et al. The CCC2000 birth cohort study of register-based family history of mental disorders and psychotic experiences in offspring. Schizophr Bull. 2015;41(5):1084-1094.
18. Nuhu FT, Eseigbe EE, Issa BA, Gomina MO. Strong family history and early onset of schizophrenia: about 2 families in Northern Nigeria. Pan Afr Med J. 2016;24:282.

19. Pakhomova SA, Korovaitseva GI, Monchakovskaia M, Vil'ianov VB, Frolova LP, Kasparov SV, Kolesnichenko EV, et al. [Molecular-genetic study of early-onset schizophrenia]. Zh Nevrol Psikhiatr Im S S Korsakova. 2010;110(2):66-69.

20. Vyas NS, Hadjulis M, Vourdas A, Byrne P, Frangou S. The Maudsley early onset schizophrenia study. Predictors of psychosocial outcome at 4-year follow-up. Eur Child Adolesc Psychiatry. 2007;16(7):465-470.

21. Gelder M, Harrison P, Cowen P. Factors predicting the outcome of schizophrenia. In: Shorter Oxford Textbook of Psychiatry. 5th Edition. New York: Oxford University Press Inc. 2008:295.

22. Rabinowitz J, Werbeloff N, Caers I, Mandel FS, Stauffer V, Menard F, Kinon BJ, et al. Negative symptoms in schizophrenia - the remarkable impact of inclusion definitions in clinical trials and their consequences. Schizophr Res. 2013;150(2-3):334-338.

23. Tsuang MT. Genotypes, phenotypes, and the brain. A search for connections in schizophrenia. Br J Psychiatry. 1993;163:299-307.

24. Hollis C. Adult outcomes of child- and adolescent-onset schizophrenia: diagnostic stability and predictive validity. Am J Psychiatry. 2000;157(10):1652-1659.

25. Bersani G, Orlandi V, Kotzalidis GD, Pancheri P. Cannabis and schizophrenia: impact on onset, course, psychopathology and outcomes. Eur Arch Psychiatry Clin Neurosci. 2002;252(2):86-92.

26. Compton WM, 3rd, Cottler LB, Ben Abdallah A, Phelps DL, Spitznagel EL, Horton JC. Substance dependence and other psychiatric disorders among drug dependent subjects: race and gender correlates. Am J Addict. 2000;9(2):113-125.

27. Kavanagh DJ, Waghorn G, Jenner L, Chant DC, Carr V, Evans M, Hemnan H, et al. Demographic and clinical correlates of comorbid substance use disorders in psychosis: multivariate analyses from an epidemiological sample. Schizophr Res. 2004;66(2-3):115-124. 